\title{
Ring-like features around young B stars
}

\author{
M. S. N. Kumar ${ }^{1}$, T. Velusamy ${ }^{2}$, C. J. Davis ${ }^{3}$, W. P. Varricatt ${ }^{3}$, and L. K. Dewangan ${ }^{4}$ \\ 1 Centro de Astrofísica da Universidade do Porto, Rua das Estrelas, 4150-762 Porto, Portugal \\ e-mail: nanda@astro.up.pt \\ 2 Jet Propulsion Laboratory, California Institute of Technology, 4800 Oak Grove Drive, Pasadena, CA91109, USA \\ 3 Joint Astronomy Center, 660 N. Aohoku Place, Hilo, HI 96720, USA \\ 4 Physical Research Laboratory, Ahmedabad-380009, India \\ Received 13 July 2009 / Accepted 19 May 2010
}

\section{ABSTRACT}

\begin{abstract}
Aims. We investigate the nature of two young intermediate mass stars namely IRAS20293+3952 (I20293) and IRAS05358+3843 (I05358), which display clearly defined $\mathrm{H}_{2}$ emission rings.

Methods. High resolution deconvolution of finely sampled 3.6-8.0 $\mu \mathrm{m}$ Spitzer-IRAC images are carried out. Continuum-subtracted $2.122 \mu \mathrm{m} \mathrm{H}_{2} 1-0 \mathrm{~S}(1)$ narrow-band images of both targets and $H K$ band spectrum of two main stars in I20293 are presented. The spectral energy distibutions (SED) of the stars enclosed in the $\mathrm{H}_{2}$ rings are constructed using photometry from the literature and combining with newly obtained Spitzer-MIPS $70 \mu \mathrm{m}$ photometry. The SEDs are modelled using a grid of radiative transfer models to obtain estimates of the star, disk and envelope physical parameters.

Results. The images reveal ring-like structures surrounding isolated young B-type stars. The modelling attributes a mass and age of $\sim 7 M_{\odot}$ and $\sim 0.1 \mathrm{Myr}$, respectively, to both young stars, in agreement with other observational indicators. The HK band spectra of I20293 display the Brackett series of HI recombination lines, which appears to correspond exclusively to the young star inside this IRAS source. The ring around I20293 appears to be a single well-defined ellipse with inner parts shining brightly in the Spitzer broad band images and outer parts displaying strong $\mathrm{H}_{2}$ emission. In the source I05358, at least two ring's are found, one circumscribing the other. The emission in the Spitzer bands and the $\mathrm{H}_{2}$ emission are mostly coincident for the inner ring. The outer ring is visible partly in the Spitzer bands and partly in $\mathrm{H}_{2}$ emission. The rings in both the sources, I20293 and I05358, have diameters of $25000-30000$ AU. The envelope sizes estimated by the SED modelling are consistent with the size of the rings estimated using the imaging data. The spectrum of the $\mathrm{H}_{2}$ ring in I20293 is suggestive of fluorescent excitation. In the source I05358, the inner ring displays a combination of PAH and $\mathrm{H}_{2}$ line emission with excess PAH emission closer to the star.
\end{abstract}

Key words. stars: formation - stars: emission-line, Be - stars: rotation - HII region

\section{Introduction}

Isolated Herbig Ae/Be stars (intermediate-mass young stars) are known to be associated with extended molecular and dust continuum emission, and to have flattened and ring-shaped geometries (Dent et al. 2005; Pantin et al. 2005). The molecular and the dust continuum emission of these objects are spread over spatial scales smaller than $1000 \mathrm{AU}$, and the molecular gas usually has a velocity structure that can be explained by Keplerian rotation (Waters \& Waelkens 1998; Alonso-Albit et al. 2009). One extreme example of a B8 type star, namely Z CMa (Herbig Be), has a very large (radius $23000 \mathrm{AU}$ ), highly flattened envelope detected by the sub-millimetre continuum emission (Waters \& Waelkens 1998).

On the other hand, massive protostars are known to be surrounded by large (10000-25000 AU) and massive (100-400 $M_{\odot}$ ) rotating toroids. There is ample evidence of these toroids, based on high resolution millimetre interferometric observations in the line and continuum (Furuya et al. 2008; Beltrán et al. 2004). Toroids are observed as extended rotating structures oriented perpendicular to known outflows from massive protostellar objects.

There is much ambiguity about the origins of massive protostellar toroids, their stability, and the large envelopes and rotational velocities of $\mathrm{HAe} / \mathrm{Be}$ stars. However, the existence of large structures which are possibly rotating is clear, based on the observational data sets as cited above. In this paper, we discuss ring-like structures around two intermediate-mass young stars found in the vicinity of well-studied massive protostellar candidates. These rings are similar in size to the toroids found around the massive protostellar candidates, but the central stars are found to be intermediate-mass young stellar objects.

Kumar et al. (2002) conducted a narrow-band $\mathrm{H}_{2}$ imaging study of a small sample of seven high-mass protostellar objects (HMPO) and discovered ring-like features around two intermediate stars in those regions. These targets are the infrared stars that coincide with the far-infrared peak of HMPO candidate IRAS 20293+3952 (hereafter I20293) and the $12 \mu \mathrm{m}$ peak of IRAS05358+3543 (hereafter I05358). I20293 is located in the Cygnus- $\mathrm{X}$ region. The estimated near and far distances to this source are $1.3 \mathrm{kpc}$ and $2 \mathrm{kpc}$, respectively, resulting in farinfrared luminosities of 2500-6300 $L_{\odot}$ (Sridharan et al. 2002). I05358 (also known as S233IR ) is located at a resolved distance of $1.8 \mathrm{kpc}$ and has a bolometric luminosity of $6300 L_{\odot}$. The FIR peak in I20293 coincides with two bright stars visible longward of $1 \mu \mathrm{m}$. Kumar et al. (2002) referred to these stars as IRS1 and IRS2. In I05358, the IRAS $12 \mu \mathrm{m}$ peak is different from the IRAS $100 \mu \mathrm{m}$ peak. The $12 \mu \mathrm{m}$ peak coincides with a bright star visible longward of $1 \mu \mathrm{m}$, which we shall call IRS1 here, whereas, the $100 \mu \mathrm{m}$ peak coincides with a cluster of millimetre sources that Beuther et al. (2002) referred to as I05358-mms. The $\mathrm{H}_{2}$ ring-like features are found around the bright infrared stars I20293-IRS1 and I05358-IRS1. These two stars appear to be relatively evolved and much of the 


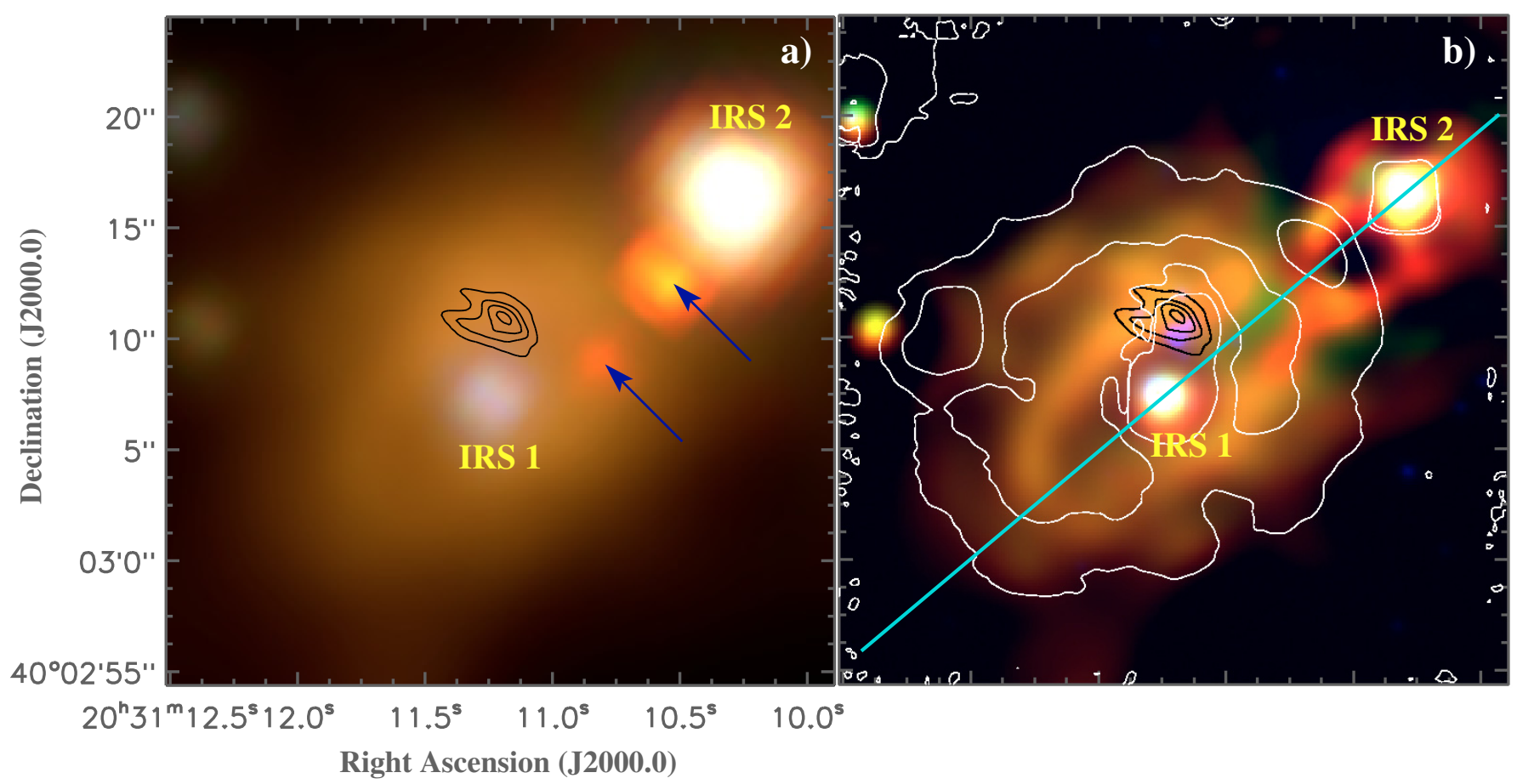

Fig. 1. a) RGB image with red $(8.0 \mu \mathrm{m})$, green $(5.8 \mu \mathrm{m})$, and blue $(4.5 \mu \mathrm{m})$ components of $\mathrm{I} 20293$ region made using short-exposure mosaic images. The arrows mark the bandwidth artifacts caused by IRS2. b) Hi-Res deconvolved results of the same region. The RGB image is obtained by coding the Hi-Res deconvolved IRAC $8.0 \mu \mathrm{m}, 5.8 \mu \mathrm{m}$ images, and near-infrared $K$-band $(2 \mu \mathrm{m})$ images as red, green, and blue, respectively. White contours ( $15 \sigma$ and $60 \sigma$ levels above the mean background) show the continuum-subtracted $\mathrm{H}_{2}$ narrow-band emission at $2.12 \mu \mathrm{m}$. The line marks the position of the slit used to obtain the near-infrared spectra. Black contours (levels are $0.10,0.13,0.15$, and $0.17 \mathrm{mJy}$ ) display the $2 \mathrm{~cm}$ continuum data from Beuther et al. (2004) representing the UCHII region.

current star-formation activity is known to occur a few tens of arcseconds away, where most of the millimetre cores are found (see Beuther et al. 2004; Palau et al. 2007). Both the millimetre dust-continuum emission and molecular lines are very weak around the stars surrounded by $\mathrm{H}_{2}$ rings. Deeper narrow-band imaging of these two sources show the $\mathrm{H}_{2}$ rings with greater depth and clarity (see Fig. 1 of Bally 2008; Beuther et al. 2004). The Spitzer Space Telescope (SST) IRAC bands are dominated by emission from very small grains (VSG) (Wood et al. 2008), polycyclic aromatic hydrocarbons (PAH) (Povich et al. 2007; Velusamy \& Langer 2008), and several $\mathrm{H}_{2}$ lines arising in warm molecular gas and shocked gas (Neufeld \& Yuan 2009). Thus, with the aid of Spitzer IRAC images and Hi-Res processing (Velusamy et al. 2008), we discovered well-defined ring features around I20293-IRS1 and I05358-IRS1. Here, we present reprocessed Spitzer data and new near-infrared data of these two sources and use them to infer some properties of the ring-like features and their central stars.

\section{Observations and data analysis}

The SST IRAC observations for I20293 and I05358 were obtained from Spitzer Science Center (SSC) archives. These data were originally observed through the astronomical observation request (AOR) number P03528 (P.I: Qizhou Zhang). The pipeline-processed basic calibrated data (BCD) files were obtained from SSC and were reprocessed using Mopex (provided by SSC) and Hi-Res deconvolution software (Backus et al. 2005; Velusamy et al. 2008). The IRAC data were taken in high dynamic range (HDR) mode. In the long exposure BCDs of the target I20293, both IRS1 and IRS2 were saturated. Figure 1a shows the mosaic image compiled using only the short exposure
BCDs. In this three-colour representation, IRAC $4.5 \mu \mathrm{m}, 5.8 \mu \mathrm{m}$, and $8.0 \mu \mathrm{m}$ images are coded by blue, green, and red, respectively. To bring out the low intensities more clearly, we used the long exposure BCDs for our Hi-Res reprocessing. All the saturated pixels in the long exposure BCDs were replaced with the corresponding values from the short exposure BCDs. In Fig. 1a, the arrows indicate the artifacts in the $5.8 \mu \mathrm{m}$ and $8.0 \mu \mathrm{m}$ images, caused by the bandwidth artifacts in these Spitzer IRAC bands (cf. Spitzer IRAC data hand Book: Sect. 3.3.1). These are due to the brightest source IRS2, which appears to be close to saturation even in the short exposure BCDs. This artifact manifests itself as a trail of bright pixels 4,8 , and 12 to the right of the triggering pixel in the detector array. This usually happens only in the long exposure BCDs and is corrected by replacing the intensities with those of the corresponding pixels in the short exposure BCD. However, such a correction of the long exposure data is not possible here, because the short exposure pixel itself is affected by this artifact. The bright point-like sources indicated by the arrows (Fig. 1a) in the case of I20293 are indeed artifacts, as their orientation with respect to IRS2 changed with rotation of the detector array during another IRAC observation (the data-set from Joseph Hora's Cygnus- $X$ survey that covers this region). Furthermore, they are artifacts because they do not have point source responses. Therefore, the intensities at these points in each BCDs were corrected by replacing them with interpolated values from the neighbouring clean pixels. The corrected BCDs were used along with the long exposure BCDs in all additional reprocessing and for Hi-Res deconvolution. The BCD data was also processed for jail-bar removal, muxbleed correction before applying the Hi-Res deconvolution (Backus et al. 2005; Velusamy et al. 2008). The point response functions (PRFs) obtained by SST inflight observations and provided by 
the SSC were used in Hi-Res. The deconvolution process was carried out with 50 iterations providing a full-width half maximum of $\sim 0.5^{\prime \prime}$ and $0.7^{\prime \prime}$ in the $3.6 \mu \mathrm{m}$ and $8 \mu \mathrm{m}$ bands, respectively. The results of the Hi-Res deconvolved images are shown in Fig. 1b. The Hi-Res processing of I05358 was simpler because the short exposure BCDs were free from the effects of bandwidth artifacts.

Near-infrared imaging and spectroscopic observations were performed using the $3.8 \mathrm{~m}$ United Kingdom Infrared Telescope (UKIRT). The $H K$ band spectra of I20293 target field were obtained using the UKIRT $1-5 \mu \mathrm{m}$ Imager Spectrometer (UIST) on the night of 21 July 2005 . UIST has a $1024 \times 1024 \mathrm{InSb}$ array and a plate scale of $0.12^{\prime \prime}$ pixel $^{-1}$. The observations were obtained using a 4 pixel width, $120^{\prime \prime}$ long slit. The $H K$ grism was used, which allowed complete wavelength coverage from $1.395 \mu \mathrm{m}$ to $2.506 \mu \mathrm{m}$ with a spectral resolution of $R \simeq 500$. Exposure times per frame were set to $60 \mathrm{~s}$, and 8 exposures were obtained per slit position resulting in a total integration time of $8 \mathrm{~min}$. The slit was aligned carefully to simultaneously include the sources IRS1, IRS2, and also the region in-between. This configuration and integration times provided a $5 \sigma$ point source sensitivity of $\sim 16$ mag in the $H K$ bands. Sky subtraction was facilitated by sliding the region up and down the slit over $\sim 36^{\prime \prime}$ and subtracting pairs of frames from one another. The bright star HIP102189 (G2V) was used for telluric calibration.

NIR imaging observations of I20293 and I05358 were performed with the facility imager UFTI (HAWAII-1 $1024 \times 1024)$. The UFTI plate scale is $0.091^{\prime \prime}$ with a field of view (FOV) of $\sim 90^{\prime \prime}$. These observations using $K$ broad-band and $\mathrm{H}_{2}$ narrowband $(2.121 \mu \mathrm{m} 10 \%$ bandwidth) filters are described in Varricatt et al. (2010).

Archival Spitzer-MIPS imaging data is available for both sources. The $24 \mu \mathrm{m}$ data is saturated in both cases, but the $70 \mu \mathrm{m}$ data is useful. Post-basic-calibrated data (PBCD) from PID:3528 (PI: Qizhou Zhang) was extracted from the archive and PRF photometry was performed using the MOPEX/APEX single frame pipeline. Photometry was obtained using the recommendations of the Spitzer data analysis cookbook recipe $24^{1}$.

\section{Results}

Figure 1b displays a composite of imaging data for I20293, showing the two point sources IRS1 and IRS2 and a bright infrared ring surrounding the source IRS1. The colour image is produced by coding the IRAC $8 \mu \mathrm{m}, 5.8 \mu \mathrm{m}$, and $K$-band $(2 \mu \mathrm{m})$ images as red, green, and blue, respectively. White contours display the continuum-subtracted $\mathrm{H}_{2}$ emission from narrow-band imaging after smoothing with a box of width $0.8^{\prime \prime}$ to match the spatial resolution of Hi-Res images. The deconvolution procedure leaves some side-lobe effect around IRS2 because it is saturated in the Spitzer images. The long solid line marks the orientation of the slit used to obtain the spectra shown in Fig. 3. A three-colour composite of I05358-IRS1 is shown in Fig. 2, where the colour coding and images used are precisely the same as those of Fig. 1. The continuum-subtracted $\mathrm{H}_{2}$ emission is shown as contours. The reader may wish to compare Fig. 1 of Bally (2008) where the $\mathrm{H}_{2}$ emission is far more prominent owing to deeper Fabry-Perot imaging. In the following, we present results that characterise the central stars I20293-IRS1, I05358-IRS1, and the ring-like features, in that order.

\footnotetext{
1 (http://ssc.spitzer.caltech.edu/dataanalysistools/ cookbook/29/)
}

\subsection{The central stars}

The central stars enclosed by the rings found in both the targets, I20293 and I05358 are modelled using a SED fitting procedure (Robitaille et al. 2006) as has been widely used. The procedure of the SED fitting for massive protostellar objects was described in detail by Grave \& Kumar (2009). We follow the same procedures and analysis to derive some physical characteristics of the central stars I20293-IRS1 and I05358-IRS1. The stars I20293IRS 1 and I05358-IRS1 were modelled using the SED fitting tool of Robitaille et al. (2007). The SEDs of both sources were constructed by using the near-infrared $J, H, K$ data from 2MASS catalogues, the Spitzer IRAC photometry from Qiu et al. (2008) and our MIPS photometry for the $70 \mu \mathrm{m}$ band. A lower limit $(0.626 \mathrm{mag})$ to the MIPS $24 \mu \mathrm{m}$ band was used because the sources are heavily saturated. The MSX Band C and E fluxes and the USNO $R$ and $I$ band magnitudes were used as upper limits for both the sources. Both I20293-IRS1 and I05358-IRS1 are detected in the USNO $R$ and $I$ bands as possible point sources that show a positional uncertainty of $2^{\prime \prime}-3^{\prime \prime}$ with respect to the sources under consideration. In comparison to clearly identified optical stars, these positional uncertainties are significant. In addition, both sources are poorly defined point sources that more closely resemble nebulosities. Therefore, the USNO photometry is used only as upper limits to the SED fitting. In the case of I20293-IRS1, additional upper limits were set from the IRAS $60 \mu \mathrm{m}$ and $100 \mu \mathrm{m}$ data of the point source catalogue. The aperture sizes used for 2MASS and Spitzer-IRAC fluxes are 1.2" and 2.4", respectively. An aperture size of 30" was used for the Spitzer-MIPS $70 \mu \mathrm{m}$ flux. The target regions shown in Figs. 1 and 2 do not contain any millimetre sources. A conservative error of $10 \%$ was assumed for all fluxes, although the true errors are smaller than the assumed values. The observed SED is scaled to match the models in the grid by using a range of values in extinction and distance. For this purpose, a range of $A_{v}=5-30 \mathrm{mag}$ was used for both the sources. The input distance range used for I20293 is 1-3 kpc for I20293 based on the known near and far distance measurements. I05358 is located at a resolved distance of $1.8 \mathrm{kpc}$, and we used a range of $1.5-2 \mathrm{kpc}$ as input to the SED fitter. This margin of $0.2-0.3 \mathrm{kpc}$ is required to account for errors in the distance determination and achieve an accurate scaling of the models from the grid. The weighted mean (weights being the inverse of $\chi^{2}$ ) of each physical parameter was computed for all the models that satisfied the criteria with $\chi^{2}-\chi_{\text {best }}^{2}<3$ where the $\chi^{2}$ is per data point.

Figure 3 shows the SEDs and radiative transfer fitted models of I20293-IRS1 and I05358-IRS1. The black dots are the data points (with $10 \%$ errors), and the downward pointing triangles are upper limits and upward pointing triangles are lower limits. The dashed line represents the assumed photosphere in the model fitting, the solid line the best-fit model, and the grey lines are the group of models satisfying the criteria $\chi^{2}-\chi^{2}$ best $<3$. The detailed physical parameters of these young stars are listed in Table 1 . The errors quoted in Table 1 correspond to the spread in the parameters of the models shown using the grey lines in Fig. 3. The data points in the $1-8 \mu \mathrm{m}$ region correspond to point sources (at a spatial resolution of better than $2^{\prime \prime}$ ), representing the stars and containing most of the photospheric and disk emission. We first note that these SEDs cannot be modelled as extincted photospheres. The SEDs of both the sources are fitted well with star+disk+envelope models. Since the fitting procedure works by scaling the models in the grid to match the observed SEDs, the most reliable output parameters are the luminosity and the shape. The data points shortward of $10 \mu \mathrm{m}$ 
Table 1. Results from SED modelling.

\begin{tabular}{|c|c|c|c|c|c|c|c|c|c|}
\hline Name & $\begin{array}{l}M_{*} \\
M_{\odot}\end{array}$ & $\begin{array}{c}R_{*} \\
\log \left(R_{\odot}\right)\end{array}$ & $\begin{array}{c}T_{*} \\
\log (K)\end{array}$ & $\begin{array}{c}\dot{M}_{\text {env }} \\
\log \left(M_{\odot} \mathrm{yr}^{-1}\right)\end{array}$ & $\begin{array}{c}M_{\text {disk }} \\
\log \left(M_{\odot}\right)\end{array}$ & $\begin{array}{c}\dot{M}_{\text {disk }} \\
\log \left(M_{\odot} \mathrm{yr}^{-1}\right)\end{array}$ & $\begin{array}{l}\text { Rout }_{\text {env }} \\
\text { AU }\end{array}$ & $\begin{array}{c}\text { Rout }_{\text {disk }} \\
\text { AU }\end{array}$ & $\begin{array}{l}\operatorname{Rin}_{\text {env }} \\
\text { Rsub }^{*}\end{array}$ \\
\hline $\mathrm{I} 2$ & $8+0$ & $12+03$ & & $-3.9 \pm 1.2$ & $-22+13$ & $-7.6 \pm 1.5$ & $37682 \pm 2$ & $117 \pm 3$ & $1 \pm 1$ \\
\hline I05358-IRS 1 & $8 \pm 0.3$ & $1.2 \pm 0.2$ & $3.8 \pm 0.1$ & $-4.8 \pm 0.8$ & $-2.1 \pm 0.5$ & $-7.5 \pm 0.7$ & $24731 \pm 2$ & $101 \pm 2$ & $4 \pm 1$ \\
\hline
\end{tabular}

Notes. ${ }^{(*)}$ Rsub is the dust destruction radius, common to both the disk and envelope. See Robitaille et al. (2006) for details.

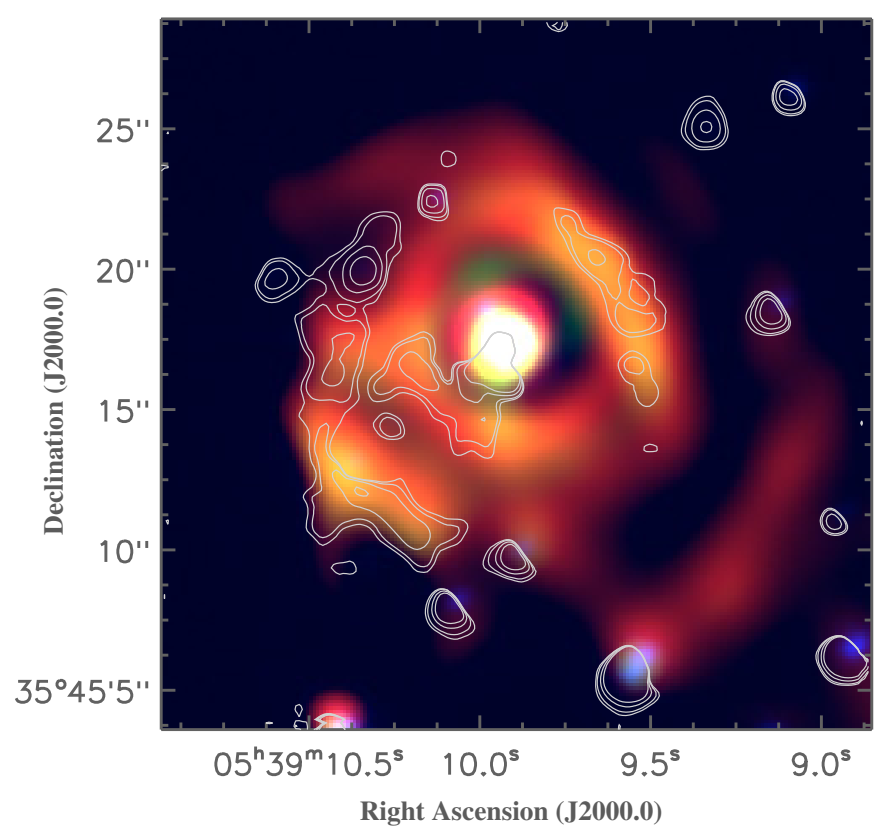

Fig. 2. Ring feature in IRAS05358+3843-IRS1. The colour coding of the image is as mentioned in Fig. 1b. White contours display continuum-subtracted $\mathrm{H}_{2}$ narrow-band emission at $2.12 \mu \mathrm{m}$. The displayed contours are at 10,15,30, and $50 \sigma$ above the mean background.

constrain the presence or absence of the disk (by the way that the total emission deviates from the stellar photospheric emission), and the data longward of $10 \mu \mathrm{m}$ constrains the envelope emission. This can be visualised in Fig. 4 (online only), where the contribution of the disk (green line) and the envelope (red line) are shown for the best-fit model of each source. However, we note that the current model grid does not take into account the contribution of continuum emission from $\mathrm{PAH}$ and small grains. Therefore for relatively hot stars, such as the sources described here, the mid-IR excess attributed to the disk is probably overestimated. The outflow cavity angles assumed in the models generally increase with age, and are based on observations of Class 0 and I protostars. This is yet another uncertain factor for intermediate mass objects such as the targets studied here. The mass and evolutionary age of the star are uniformly sampled in the model grid. This allows ourselves to identify models with a unique range of mass and age that most closely represent the observed data. Subsequently, the radius and temperature of the star are interpolated using evolutionary models. The targets studied here are modelled as embedded/disk objects. In Fig. 3, we note that some of the observed data points actually trace the dashed curve (input photosphere). This agrees with the imaging data that display bright infrared stars with low extinction. The models place the mass and evolutionary age of both the sources roughly at $6.8 M_{\odot}$ and $0.1 \mathrm{Myr}$, respectively. Many of the stellar properties are similar between the two sources and differ only for the envelope and disk properties.

The envelope and disk accretion rates here are relatively low compared to the typical accretion rates found in objects that are actively accreting and associated with millimetre cores (see Grave \& Kumar 2009). However, we note that the size of the modelled envelopes are a few tens of thousands of AU, similar to the projected size of the ring-like features (see next section). We emphasize that the definition of the envelope does not indicate that clearly defined geometric structures are present. They merely represent the distance $(\sim 1000-100000 \mathrm{AU})$ at which the optically thin radiative temperature falls to $30 \mathrm{~K}$ (Robitaille et al. 2006).

Figure 4 shows the $H K$ band spectra of the I20293 region encompassing the stars IRS1, IRS2, and the $\mathrm{H}_{2}$ ring feature. No spectra are available for I05358-IRS1. The spectra of IRS1 and IRS2 in I20293 shows the Brackett series hydrogen recombination line emission (spread between 1.5-2.2 $\mu \mathrm{m}$ ) and part of the Pfund series (after $2.4 \mu \mathrm{m}$ ). The long slit encompasses the entire infrared nebula. We thus integrated the emission along the full length of the slit, excluding the stars IRS1 and IRS2 to find only trace amounts of $\mathrm{Br} \gamma$ line emission (the brightest of the observed HI series). In contrast, the stars themselves show intense $\operatorname{Br} \gamma$ emission, imply that the observed ionised emission is localised in the true stars IRS1 and IRS2 and is insignificant in the extended infrared nebula. The region between the stars IRS 1 and IRS2 showed more $\mathrm{H}_{2}$ emission than $\mathrm{Br} \gamma$, which is discussed in the next section. The diagnostic information derived from the $H K$ band spectra that IRS1 and IRS2 are young intermediatemass stars capable of producing compact HII regions is therefore in agreement with the results of the SED modelling.

In Fig. 1b (I20293-IRS1), the blue stars ( $K$-band) located in the inner rim of the ring feature, coincide with the $2 \mathrm{~cm}$ continuum emission mapped by the VLA (Beuther et al. 2004; Palau et al. 2007). This group actually consists of five faint stars that are clearly detected in the sub-arcsec resolution $K$-band image (see Varricatt et al. 2010). The VLA emission is not associated with IRS1. The spatial resolution of both the VLA data and our $K$-band data is better than $0.5^{\prime \prime}$, thus confirming this non-association. It is not possible to ascertain whether the five faint stars associated with the VLA emission are actually located within the ring-feature, as is apparent from the projected image. If they are indeed located within the ring, then it forms an interesting case of a "circumcluster ring". We can only conclude that these stars are a loose association or micro-cluster located in the larger infrared nebula.

\subsection{The ring features}

The ring-like features around I20293-IRS1 and I05358-IRS1 can be seen in Figs. 1 and 2 from the infrared emission in the high-resolution processed images and the overlaid continuumsubtracted $\mathrm{H}_{2}$ emission contours. The infrared continuum 
M. S. N. Kumar et al.: Ring-like features around young B stars
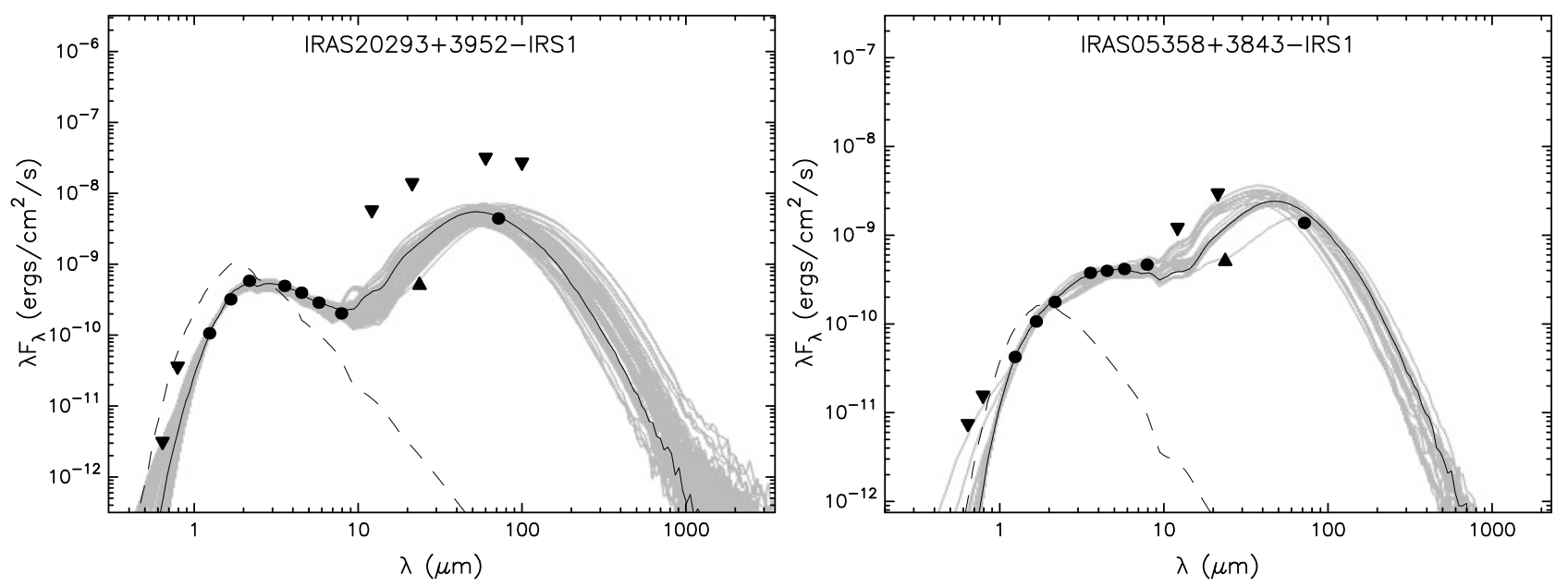

Fig. 3. Spectral energy distributions of I20293-IRS1 and I05358-IRS1 sources. Black dots shows the data points and the triangles are lower and upper limits. The dashed line represents the assumed photospheric emission in the model, the solid line is the best-fit model and the group of grey lines are the models satisfying the $\chi^{2}-\chi^{2}$ best $<3$ criteria.
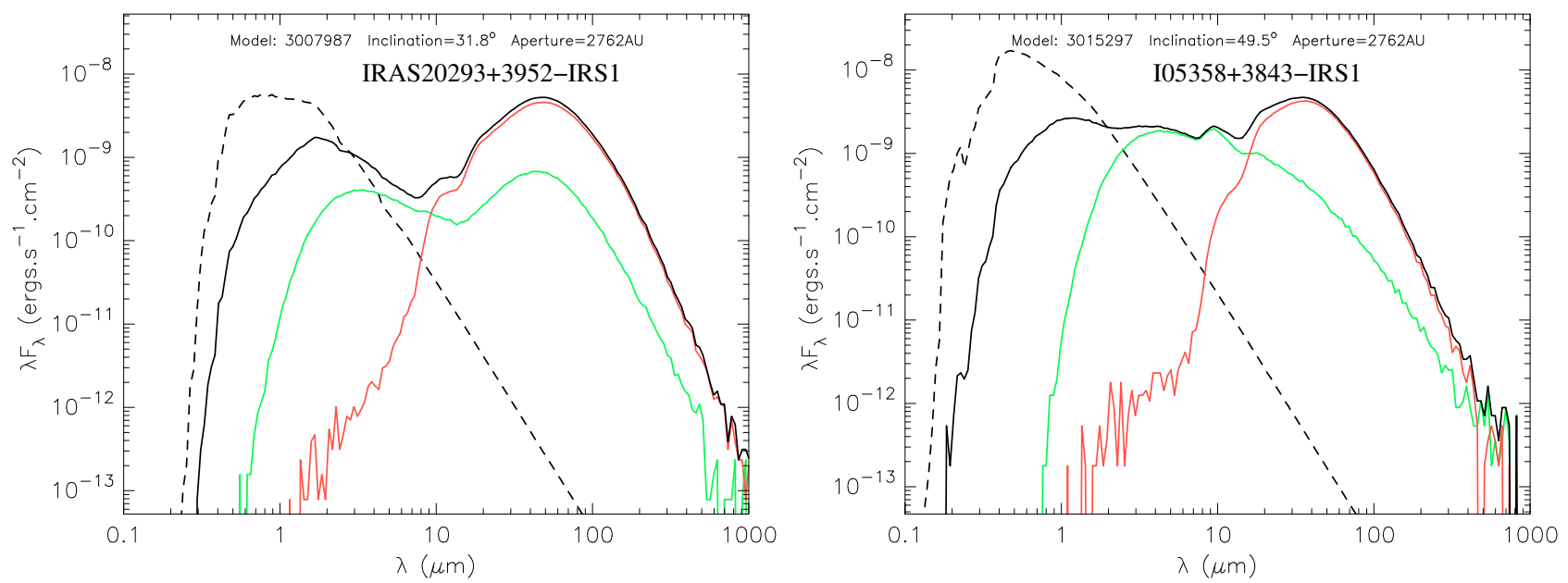

Fig. 4. Decomposition of the best-fit model (black curve). The dashed curve shows the input photosphere, green and red curves represent the disk and envelope emission, respectively.

emission traces the inner edge and the $\mathrm{H}_{2}$ contours the outer parts or shell of the rings in both cases.

I20293-IRS1: Figure 1 displays the ring around this target. Although the inner part of the ring appears as a single clearly defined ellipse, it appears to be extended, the $2.122 \mu$ m emission tracing the outer regions more prominently. The major axes of the ellipses (Spitzer-IRAC and $\mathrm{H}_{2}$ ) were measured in the images to calculate the projected diameters at a distance of $2 \mathrm{kpc}$. We obtained intensity cuts along the axes and used the peak-to-peak distance to measure the size. The projected mean radii of the IRAC and $\mathrm{H}_{2}$ parts of the rings are $\sim 7000 \mathrm{AU}$ and $\sim 24000 \mathrm{AU}$ $(0.11 \mathrm{pc})$, respectively. Assuming that these rings are circular in nature, its projected appearance as an ellipse allows us to calculate the inclination angle, which is the inverse cosine of the ratio of the minor to major axis. This turns out to be roughly $60^{\circ}$ in this case. The mean inclination angle and the spread from the SED fitting is $31 \pm 14^{\circ}$. Robitaille et al. (2007) argue that the SED fit is effective in representing the evolutionary stages, but the inclination to the line of sight in most cases is not well determined (see Sect. 3.2.1 of their paper for a discussion). The spectrum of the $\mathrm{H}_{2}$ ring shown in Fig. 4, allows us to obtain a rough estimate of the ratios of the $\mathrm{H}_{2} 1-0 \mathrm{~S}(1)$ and $\mathrm{H}_{2}$ 2-1 S(1) lines. This ratio $(0.49)$ is consistent with fluorescent emission rather than shocked (required ratio is $\sim 0.2$ ) emission.

I05358-IRS1: Figure 2 shows the ring surrounding this source. The ring appears relatively circular, with the IRAC band emission coinciding with the $\mathrm{H}_{2}$ emission. Unlike I20293-IRS1, this source is surrounded by multiple rings, with evidence of at least two and possibly three (see the outermost partial ring to the bottom right corner of Fig. 2). The $\mathrm{H}_{2}$ emission contours surrounding blue stars are residues resulting from the subtraction of the narrowband from the continuum image. The inner ring in this source has a radius of $\sim 12600 \mathrm{AU}$ and the outer (partial second ring) ring has a radius of $\sim 15000 \mathrm{AU}$. The partial ring that could be called the third segment lying to the bottom right corner of the image, is at a radius of $\sim 20000 \mathrm{AU}$. The inclination angle and spread from SED fitting for this source is $47 \pm 16^{\circ}$. The lack of clearly defined ellipse in this case does not allow us to measure the inclination angle to be the inverse cosine of axis ratio.

Given the absence of available mid-infrared spectra for these sources, it is not straightforward to conclude the nature of emission from the rings. Nevertheless, differences between the morphological appearances in different IRAC bands can help 


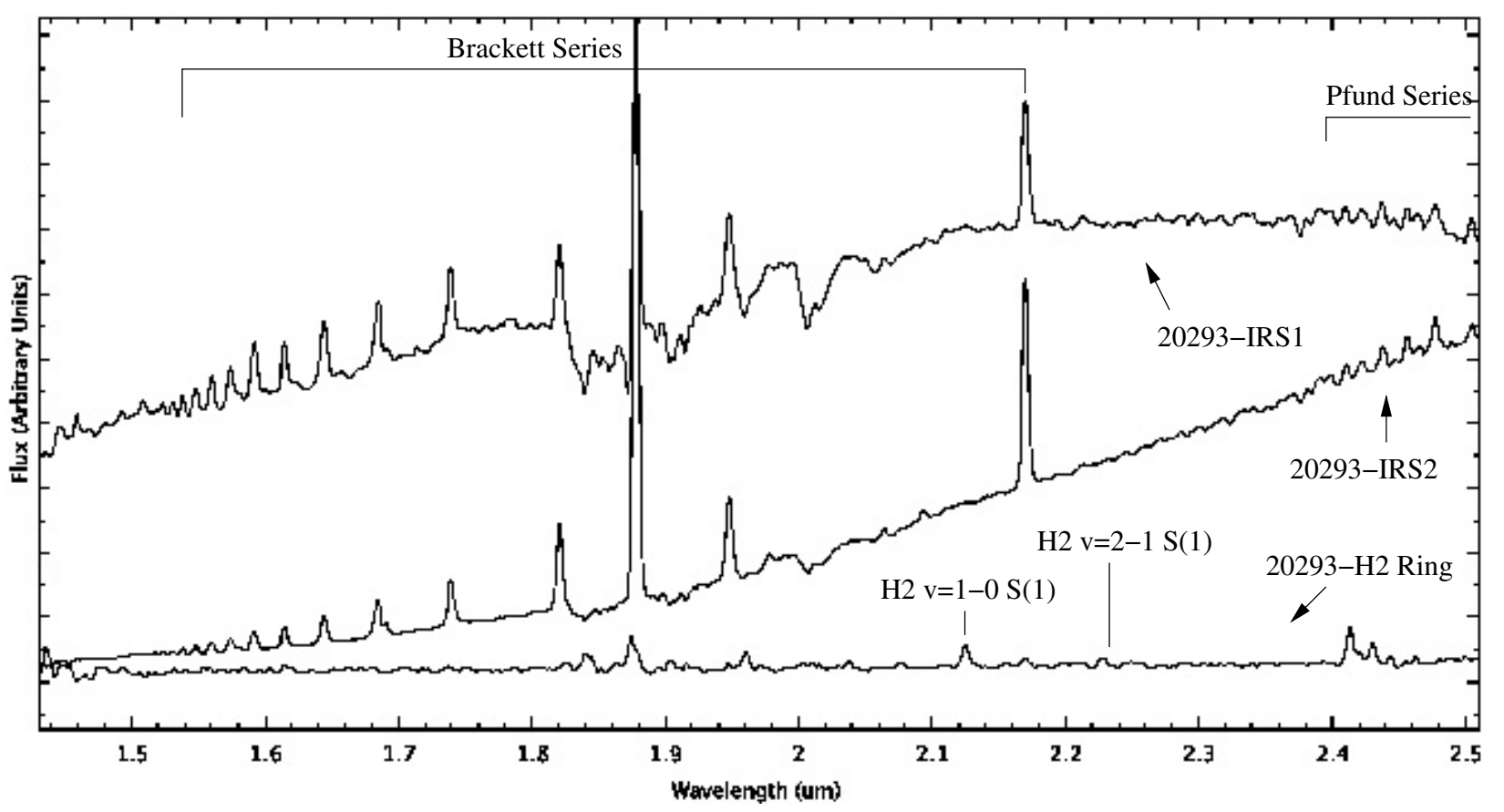

Fig. 5. HK band spectra of I20293-IRS1, I20293-IRS2, and the I20293-H2-torus.

determine the origin of the emission. Polycyclic aromatic hydrocarbons (PAH), very small grains (VSGs), and $\mathrm{H}_{2}$ rotationalvibrational emission lines are major contributors to the IRAC bands. The PAH/VSG emission is easily produced in the vicinity of a UV source in the interstellar medium, whereas $\mathrm{H}_{2}$ emission lines are produced in shocked and/or fluoresced dense gas. The PAH bands are found only in Ch1 $(3.6 \mu \mathrm{m}), \mathrm{Ch} 3(5.8 \mu \mathrm{m})$, and Ch4 $(8.0 \mu \mathrm{m})$ of the IRAC bands, and have their maximum contributions in $\mathrm{Ch} 3$ and $\mathrm{Ch} 4$ bands. The IRAC Ch2 $(4.5 \mu \mathrm{m})$ band does not include any $\mathrm{PAH}$ emission, therefore ensuring that it is an excellent "reference band" for distinguishing continuum from PAH emission. Povich et al. (2007) demonstrated that dividing both the $\mathrm{Ch} 4$ and $\mathrm{Ch} 3$ images (containing PAH lines) by the $\mathrm{Ch} 2$ image (absence of PAH) can be an excellent means of identifying the PAH emission. However, this technique is not useful if the targets have shocked emission that appears bright in the IRAC Ch2 band. By using Spitzer IRS spectra, they demonstrate how the results from this simple technique represents the true PAH emission in the star-forming region M 17. Yet another property of PAH emission is that the bands are excited easily, and the PAH relative intensities are quite insensitive to the illuminating source flux (Wood et al. 2008).

Emission from molecular hydrogen is spread across all four IRAC bands. The $\mathrm{Ch} 2$ band is popular for identifying jets/outflows, and $\mathrm{Ch} 3$ and $\mathrm{Ch} 4$ are also known to have important contributions from emission lines of shocked $\mathrm{H}_{2}$ (Neufeld \& Yuan 2009).

Based on the above findings, we applied the method of Povich et al. (2007) of dividing the Ch3 and Ch4 images by the Ch2 image for both the sources I20293 and I05358. Given that the IRAC Ch2 represents continuum and/or shocked emission while $\mathrm{Ch} 3$ and $\mathrm{Ch} 4$ are dominated by $\mathrm{PAH}$ emission, any positive emission in the divided images will indicate excess PAH emission. Among the two sources studied here, the division of the I05358 images resulted in positive emission distributed in a partial ring tracing the inner edge of the first (innermost) ring. There was no significant positive emission in the source
I20293-IRS1. Therefore, in the source I05358-IRS1 the emission from the first ring is composed of a combination of PAH and $\mathrm{H}_{2}$ emission, but with an excess PAH emission closer to the central star. This is similar to the case of NGC 2316 for which Velusamy \& Langer (2008) detected a $\mathrm{H}_{2}$ ring and intense PAH emission within the $\mathrm{H}_{2}$ ring around the young $\mathrm{B} 3$ star.

\section{Discussion}

The observational data that we have presented here demonstrates that ring-like structures exist around intermediate-mass stars of $\sim 7 M_{\odot}$, at an evolutionary age of $\sim 0.1 \mathrm{Myr}$. Thus, these stars are at the end of the star formation phase if we assume that the typical timescale for massive star formation (e.g., McKee \& Tan 2003 ) is 0.1 Myr. The lack of significant dust continuum and gas emission around the stars and their appearance in the optical $\mathrm{R}$ bands also indicate that they are probably at the end of the star formation phase. The accretion rates and disk masses obtained from SED modelling, that are comparatively low, are also consistent with their evolved nature. Young B stars, such as these, are well known to produce compact ionised regions, which, in the case of I20293-IRS1, are clearly present because of the observed HI recombination lines in the NIR spectra. The localised emission of the HI lines in I20293-IRS1 also suggests that the UV from the central star has not ionised the surrounding cloud and destroyed the molecular gas. The extended $\mathrm{H}_{2}$ emission from narrow-band imaging and the $\mathrm{H}_{2}$ lines in the spectra are proof that molecular material survives in the ring-like structures around the central B star. These evolutionary signatures, or, the age computed by the SED model, imply that these structures have remained stable for at least $10^{5}$ years.

If we assume that these intermediate mass stars form in similar ways to low mass stars, the observations presented here provide a few clues to the possible nature of the circumstellar environment:

1) in the case of I20293-IRS1, the localised HI line emission is indicative of significant UV radiation close to the stars, 
but, the modelled temperatures $(\sim 6000 \mathrm{~K})$ are not consistent with this claim. We note that the SED model grid is produced by uniformly sampling the mass and age (Robitaille et al. 2006) and subsequently applying the pre-main-sequence evolutionary models (Bernasconi \& Maeder 1996; Seiss et al. 2000) to obtain consistent radius and temperature. Although Robitaille et al. (2007) demonstrate that this method reproduces meaningful temperatures for low mass stars, the case of intermediate and massive stars are debatable and are strongly influenced by the existing evolutionary models. In the case of young low-mass stars, strong UV radiation is produced in a magnetosphere, indicated by several emission lines, including the $\mathrm{Br} \gamma$ emission, commonly observed in young T-Tauri type stars. It is possible that the UV radiation in the B stars is produced by accretion activity in the close environment of the stars. The lower photospheric temperatures of the B type stars here may only be consistent with large radii, which are predicted by many evolutionary models (e.g. Bernasconi \& Maeder 1996; Seiss et al. 2000; Hosokawa \& Omukai 2009) and also found in a study of a larger sample of massive protostellar objects by Grave \& Kumar (2009). The mass-radius relationships of most evolutionary models imply that there is some bloating of the protostar, typically in the mass range 4-10 $M_{\odot}$ and accretion rates in the range $10^{-5}-10^{-3} M_{\odot} \mathrm{yr}^{-1}$ (Palla \& Stahler 1993; Hosokawa \& Omukai 2009);

2) even if the UV radiation produced close to the star is significant, two possible effects may permit the survival of molecular gas around these young stars: a) the UV radiation may be effectively channelled out in the outflow cavities (Krumholz et al. 2005) during much of the early star-formation phase; and b) an appropriate inclination angle and a thick disk can cause a shadowing effect to protect the molecular material from exposure to destructive radiation in the outer envelope. However, the UV flux shining on this molecular material can produce the observed fluorescent $\mathrm{H}_{2}$ emission;

3) finally, the ring-like structures may reflect the stability provided by rotation to the circumstellar matter around intermediate mass stars. Toroids of large dimensions such as a few tens of thousands of AU imply rotational stability and support in intermediate and massive star-forming regions. For example, de Wit et al. (2009) detect large envelopes around several young massive stars, using high resolution $24 \mu \mathrm{m}$ observations. These envelopes are found to have flat radial density profiles in the inner $1000 \mathrm{AU}$, which are indicative of rotational support in massive protostars. The envelopes/toroids found by Furuya et al. (2008) are examples of these flattened structures at very early stages, the observations of de Wit et al. (2009) being example of intermediate stages and the ring-like features discussed here probably representing the fossil remains. Only the size of the rings from this study are similar to the sizes of the toroidal structures found around massive protostellar objects. Detailed kinematic data will be needed to prove whether there are yet more similarities (such as in rotational velocity). Nevertheless, the ring features may not be a surprise if we assume that regions such as these can produce $\mathrm{HAeBe}$ stars or even classical Be stars, which are known to have large rotational velocities (Waters \& Waelkens 1998; Porter \& Rivinius 2003).

Acknowledgements. Our sincere thanks to Henrik Beuther for providing the millimetre and VLA data of these sources that is used for analysis. Kumar is supported by a Ciência 2007 contract, funded by FCT/MCTES (Portugal) and POPH/FSE (EC) and a research grant PTDC/CTE-AST/65971/2006 approved by the FCT (The Portuguese national science foundation). This work is based [in part] on observations made with the Spitzer Space Telescope, which is operated by the Jet Propulsion Laboratory, California Institute of Technology under a contract with NASA.

\section{References}

Alonso-Albi, T., Fuente, A., Bachiller, R., et al. 2009, A\&A, 497, 117

Backus, C. R., Velusamy, T., Thompson, T. J., \& Arballo, J. K. 2005, in ADASS XIV, ed. P. L. Shopbell, M. C. Britton, \& R. Ebert (San Francisco, CA: ASP), ASP Conf. Ser., 347, 61

Bally, J. 2008, ed. H. Beuther, H. Linz, \& T. Henning, ASP Conf. Ser., 387, 158 Beltrán, M. T., Cesaroni, R., Neri, R., et al. 2004, ApJ, 601, L187 Bernasconi, P. A., \& Maeder, A. 1996, A\&A, 307, 829

Beuther, H., Schilke, P., Sridharan, T. K., et al. 2002, A\&A, 383, 892

Beuther, H., Schilke, P., \& Gueth, F. 2004, ApJ, 608, 330

Beuther, H., Zhang, Q., Sridharan, T. K., \& Chen, Y. 2005, ApJ, 628, 800

Cesaroni, R., Codella, C., Furuya, R. S., \& Testi, L. 2003, A\&A, 401, 227

Cesaroni, R., Daniele, G., Giuseppe, L., Walmsley, M., \& Zhang, Q. 2006, Nature, 444, 703

Dent, W. R. F., Greaves, J. S., \& Coulson, I. M. 2005, MNRAS, 359, 663

de Wit, W. J., Hoare, M. G., Fujiyoshi, T., et al. 2009, A\&A, 494, 157

Furuya, R. S., Cesaroni, R., Takahashi, S., et al. 2008, ApJ, 673, 363

Grave, J. M. C., \& Kumar, M. S. N. 2009, A\&A, 498, 147

Hosokawa, T., \& Omukai, K. 2009, ApJ, 703, 1810

Krumholz, M. R., McKee, C. F., \& Klein, R. I. 2005, ApJ, 618, L33

Kumar, M. S. N., \& Grave, J. M. C. 2007, A\&A, 472, 155

Kumar, M. S. N., Bachiller, R., \& Davis, C. J. 2002, ApJ, 576, 313

McKee, C. F., \& Tan, J. C. 2003, ApJ, 585, 850

Neufeld, D. A., \& Yuan, Y. 2008, 678, 974

Pantin, E., Bouwman, J., \& Lagage, P. O. 2005, A\&A, 437, 525

Palau, A., Estalella, R., Girart, J. M., et al. 2007, A\&A, 465, 219

Palla, F., \& Stahler, S. W. 1993, ApJ, 418, 414

Povich, M. S., Stone, J. M., Churchwell, E., et al. 2007, ApJ, 660, 346

Porter, J. M., \& Rivinius, T. 2003, PASP, 115, 1153

Qiu, K., Zhang, Q., Megeath, S. T., et al. 2008, ApJ, 685, 1005

Robitaille, T. P., Whitney, B. A., Indebetouw, R., Wood, K., \& Denzmore, P. 2006, ApJS, 167, 256

Robitaille, T. P., Whitney, B. A., Indebetouw, R., \& Wood, K. 2007, ApJS, 169, 328

Seiss, L., Dufour, E., \& Forestini, M. 2000, A\&A, 358, 593

Sridharan, T. K., Beuther, H., Schilke, P., \& Menten, K. M. 2002, ApJ, 566, 931

Varricatt, W. P., Davis, C. J., Ramsay, S., \& Todd, S. P. 2010, MNRAS, 404, 661

Velusamy, T., \& Langer, W. D. 2008, ApJ, 136, 602

Velusamy, T., Marsh, K. A., Beichman, C. A., Backus, C. R., \& Thompson, T. J. 2008, ApJ, 136, 197

Waters, L. B. F. M., \& Waelkens, C. 1998, ARA\&A, 36, 233

Wood, K., Whitney, B. A., Robitaille, T., \& Draine, B. T. 2008, ApJ, 688, 1118

Zinnecker, H., \& Yorke, H. 2007, ARA\&A, 45, 481 